\title{
How Does Outcomes Research Help Advance Our Knowledge of Patient Outcomes in Hepatopancreaticobiliary Surgery?
}

\author{
Donna Marie L. Alvino ${ }^{1}$ • David C. Chang ${ }^{1}$. Zhi Ven Fong ${ }^{1}$
}

Received: 14 August 2015 / Accepted: 30 December 2015 / Published online: 9 February 2016

(C) 2016 The Society for Surgery of the Alimentary Tract

\begin{abstract}
Randomized controlled trials have historically been regarded as the gold standard of modern clinical research tools, allowing us to elucidate the efficacy of novel therapeutics in an unparalleled manner. However, when attempting to generalize trial results to broader populations, it becomes apparent that the unexplained outcome variability exists among treatment recipients, suggesting that randomized controlled trials harbor inherent limitations. Herein, we explore the benefits of health services (outcomes) research utilization in addressing variation in patient outcomes following surgical intervention in the nonrandomized setting, with a specific focus on hepatopancreaticobiliary surgery outcomes. To achieve this, we have constructed a framework that outlines the complex interactions existing between therapeutic, patient, and provider factors that subsequently lead to variation in outcomes. By exploring examples in the current literature, we have highlighted the areas where the knowledge is currently lacking and can be further strengthened through the application of outcomes research. Furthermore, we have attempted to demonstrate the utility of alternative study designs in the investigation of novel clinical questions currently unanswered in the field of hepatopancreaticobiliary surgery.
\end{abstract}

Keywords Health services research $\cdot$ Outcomes $\cdot$ Pancreas · Hepatopancreaticobiliary surgery

In the era of modern medicine, randomized controlled trials (RCTs) have played an integral role in elucidating the efficacy of breakthrough therapeutic modalities. More recently, health services (outcomes) research has risen as another form of research methodology. Outcomes research may be even more valuable in a subspecialized field such as hepatopancreaticobiliary (HPB) surgery, in which RCTs are less commonplace than in other subspecialties, likely due to lower overall incidence rates of pathology and limited availability of study participants. ${ }^{1}$ More importantly, outcomes research allows us to overcome the limitations of

Zhi Ven Fong

zfong@partners.org

1 Department of Surgery, Codman Center for Clinical Effectiveness in Surgery, Massachusetts General Hospital, Harvard Medical School, 165 Cambridge Street, Boston, MA 02114, USA
RCT design. RCTs provide unparalleled internal validity, often at the expense of external validity, and when attempting to generalize study results to a broader population, we begin to define clear knowledge gaps pertaining to the unexplained outcome variability in recipients of the same therapies. This suggests that the treatment choice is not the sole outcome determinant:

Outcome $>$ Therapy

It becomes apparent that patient outcomes are actually the product of multiple variables intrinsically linked to produce an end result, and that patient and provider qualities can also impact the outcome of a therapy. ${ }^{2}$ Simply put, therapy is but one factor in a larger equation:

Outcome $=$ Therapy + Patient + Provider

RCT-driven research is limited in its ability to simultaneously assess the impacts of all three variables on patient outcomes. Furthermore, it is difficult to appropriately randomize patients on the basis of certain individual qualities, such as age or gender, and becomes nearly impossible to randomize them to provider characteristics. This is yet another possible reason 
for the overall lack of RCTs in HPB surgery - it is exceedingly difficult to account for the substantial variability in the surgical quality or approach amongst surgeons within a properly designed RCT secondary to anatomic complexity and the potential variability in different operative stages. ${ }^{1}$

As we hope will become evident, outcomes research can uniquely allow us to appreciate how various patient and provider factors, both independently and in conjunction with therapeutic interactions, can explain the outcome variability in the non-randomized setting. Additionally, we hope to demonstrate how utilization of this framework can incite investigation of novel clinical questions currently unanswered in the field of HPB surgery. Lastly, we will discuss the utility and appropriateness of employing several alternative study designs to address such unanswered questions.

\section{Outcome $=$ Therapy + PATIENT + Provider}

The effects that patient characteristics (age, race, sex, comorbidities, socioeconomic status, etc.) can have on responses to treatment modalities have previously been studied. ${ }^{1}$ As mentioned, the primary goal of a well-powered RCT is to eliminate potential confounding effects of these variables as much as possible to solely determine the efficacy of a specific intervention within one well-defined patient population. ${ }^{3}$ By controlling for these factors, RCTs inherently presuppose that the therapeutic intervention in question is the most important determinant of patient outcomes. However, even within an RCTdefined populace, patient factors such as age, weight, and comorbidities may harbor a significant, unforeseen impact on their disease processes and subsequent outcomes, regardless of the treatments rendered. Outcomes research can help to identify these independent patient outcome determinants so as to explain the discrepancy when translating RCT results in a real-world setting. As an example, a dual-institution RCT conducted by Berger et al. compared the efficacy of two reconstructive techniques for pancreaticojejunostomy $(\mathrm{PJ})$ following pancreaticoduodenectomy (PD) for proximal pancreatic lesions in preventing the formation of postoperative pancreatic fistula (PF): patients were randomized to undergo either ductto-mucosa anastomotic reconstruction or invagination reconstruction. ${ }^{4}$ The results demonstrated a markedly higher rate of PF in the duct-to-mucosa study-arm (24\%) as compared to the invagination study-arm (12\%), thus purporting that employment of an invagination technique for PJ following PD was the optimal choice for prevention of PF. However, the study did not account for patient factors that might also directly influence the development of postoperative PF and many such factors have been documented in the literature. Lin et al., for instance, looked at numerous patient risk factors for PF formation following PD, including gender, race, and several comorbidities (hypertension, smoking, diabetes mellitus (DM), and coronary artery disease (CAD)); they found that $\mathrm{CAD}$ was an independent risk factor for developing PF on multivariable analysis. ${ }^{5}$ Separately, Relles et al. documented that patients with $\mathrm{BUN} \geq 10 \mathrm{mg} / \mathrm{dL}$ and serum albumin $<2.5 \mathrm{~g} / \mathrm{dL}$ on the first postoperative day had an $18.5 \%$ risk of $P F$ versus patients with neither risk factor (PF rate of $3.6 \%$ ). ${ }^{6}$ Taken together, it becomes evident that much more is at play in the development of postoperative PF than the surgical technique alone. The knowledge of the patient factors affecting surgical outcomes can help to guide the actions of physicians in the preoperative and perioperative period.

\section{Outcome $=$ Therapy + Patient + PROVIDER}

The term provider encompasses a system with which patients interact on multiple hierarchical levels, including hospitals, physicians, nurses, and administrators, as well as government health policy. One can imagine that there must exist a significant variability between providers with respect to the regional location, hospital teaching affiliation, and physician training and experience. Perhaps, the greatest example of provider impacts upon surgical outcomes stems from the concept of volume-outcome effect. Birkmeyer et al. documented that Medicare patients undergoing surgical intervention at low-volume institutions had significantly higher perioperative mortality rates than those seeking care at high-volume facilities, irrespective of the quality or type of operative intervention being performed. $^{7}$ While it may seem intuitively necessary to account for provider differences in the assessment of an intervention's efficacy or superiority, RCTs are limited in this capacity by design - factors such as hospital volume and physician experience are often unaccounted for and thus may produce misleading results. Referring back to Berger et al. as an example, the overall mortality for patients undergoing the more commonly performed PD with duct-to-mucosa anastomosis was $2 \%$ while the mortality rate for PD with invagination was surprisingly found to be $0 \%{ }^{4}$ Yet, the average mortality for patients undergoing PD is estimated to be closer to $4 \%$ in tertiary-care centers nationally, an apparent doubling to quadrupling in the risk of death from PD. ${ }^{8}$ The superior outcomes demonstrated by the two institutions are likely explained by their impressive pancreatectomy volume, having performed 198 resections over the 2-year trial period. This further highlights the important impact that providing institutions and surgeons have on patient outcomes. From this, it becomes obvious that outcomes of surgical interventions are highly dependent upon the experience of the provider; this holds especially true in a field as technically complex as HPB surgery. With the data that outcomes research provides, 
patients can make an informed decision to entrust their safety to the most experienced of surgeons.

\section{Patient Provider}

\section{$\downarrow \quad \downarrow$ \\ III. Outcome $\leftarrow \leftarrow \leftarrow \leftarrow \leftarrow$ Therapy}

Lastly, outcomes research can help to guide our understanding of the more intricate interactions that occur between the three primary influences of outcomes. Our seemingly simple equation evolves in complexity when considering how patients and providers can also serve in the capacity of effect modifiersthat is, patient and provider factors not only have direct impacts on outcomes of surgical interventions as previously discussed, but they also indirectly affect how treatments render certain outcomes. The investigation of such interactions has rarely been documented in the literature and as such is perhaps the most promising application of outcomes research. Assessment of these interactions can allow us to develop appropriate patient and provider indications for therapeutic interventions.

To continue with the Berger study example, patients were intraoperatively stratified by the texture of pancreatic tissue (soft versus hard) prior to the study-arm randomization in an effort to determine if pancreatic remnant texture should be considered an indication for performing a particular technique. ${ }^{4}$ This was based on the well-known association of lower rates of PF following PD reconstruction in patients with hard pancreatic remnants caused by chronic pancreatic ductal obstruction leading to distal atrophy and fibrosis. While they found higher rates of PF formation when anastomosing soft remnants versus fibrotic remnants, regardless of the technique employed (verifying pancreatic tissue texture as an independent risk factor for PF), they documented a doubling of relative risk for $\mathrm{PF}$ formation amongst both pancreatic remnant subtypes when comparing techniques. PF rates jumped from $18 \%$ with the invagination anastomosis to $36 \%$ with the duct-to-mucosa anastomosis in the soft remnant sub-group and from $6 \%$ to $11 \%$ in the hard remnant sub-group, respectively, suggesting that there was no interaction between the pancreatic remnant texture and technique. Yet, for argument's sake, if they had instead documented a constant PF rate in the hard remnant subgroup, $6 \%$ with invagination and $6 \%$ with duct-to-mucosa anastomosis, while the PF rates doubled (18 $\%$ to $36 \%$ ) for their soft remnant subgroup, this would suggest that employing the duct-to-mucosa technique actually worsens outcomes when utilized specifically in patients with soft pancreatic remnants (a relative risk of two compared to one). In that context, the pancreatic tissue texture indirectly modifies how a particular anastomosis affects the rate of PF formation. Soft pancreatic tissue texture would thus be a specific patient indication for choosing to perform an invagination technique for PJ over a duct-to-mucosa technique.

A significant question still stands to be answered: if invagination anastomosis in PJ reconstruction following PD has been shown to be superior in an RCT, why do we not see it more frequently employed amongst pancreatic surgeons? The answer again stems from the inherent issue of RCTs and the fact that they often cannot account for how provider variability indirectly influences treatment effectiveness. Berger et al. attempted to resolve the apparent discrepancy in $\mathrm{PF}$ rates among their two institutions (12\% PF rate at hospital A versus $26 \% \mathrm{PF}$ rate at hospital B) by citing a higher incidence of patients with soft pancreatic remnants at hospital B, but they also referenced an inability to account for the effects of surgeon comfort with each technique. ${ }^{4}$ Invagination was the documented default anastomotic technique at hospital A while hospital B surgeons typically employed the duct-to-mucosa method. It seems quite feasible that one or more surgeons at hospital A were particularly facile at performing an invagination PJ, but relatively less comfortable at the duct-to-mucosa method compared to their hospital B counterparts. Using the existent data to stratify surgeons by operative technique, we may learn that invagination PJ is the superior method of reconstruction only amongst hospital A surgeons, perhaps decreasing their PF rate from $19 \%$ to $5 \%$ when compared to a duct-to-mucosa approach, while the opposite may be true of hospital B surgeons. Without controlling for the potentially large confounding impact of provider skill on operative technique, the results of an RCT can be easily skewed to demonstrate the superiority of an approach that is inapplicable in a broader provider setting. Furthermore, most surgeons are taught to perform a duct-to-mucosa anastomosis as the standard method of reconstruction for PD and as such are far more skilled in this approach. To suggest that they instead attempt to utilize a foreign technique based on the results of a single RCT skewed by the mastery-level skill of a few surgeons appears somewhat irrational — outcomes research can help to elucidate these types of limitations to RCT findings.

Undoubtedly, outcomes research is a crucial alternative to RCT design given the complex interactions occurring between the various outcome-determining factors described. However, outcomes research itself encompasses a certain degree of complexity when considering the numerous alternative study designs and databases one might consider utilizing to address such clinical questions. Herein, we shall briefly describe several well-known alternative study designs and analytical methods and address the utility, appropriateness, and limitations of each in an effort to mitigate confusion and assist researchers in their outcomes research endeavors.

Propensity scoring is an analytical modality often utilized in outcomes research to account for patient factors, or covariates, that may bias selection for treatment in a nonrandomized setting. Scores are used to dictate the probability 
that patients will be treated based on an observed group of covariates. Conventional multivariable analysis is often used to address the issue of covariate confounding, but its utility is often limited when there is marked variability in the distribution of such covariates between treatment and control groups. ${ }^{9}$ Thus, propensity scoring can be used to provide greater comparability between group covariate distributions and can eliminate the extensive number of variables to be accounted for in analyses by collapsing numerous confounding covariates (age, race, Charlson comorbidity index, etc.) into one score. Furthermore, propensity score matching can be used to match treatment and control subjects and better balance the covariate distributions among study groups. For example, a study determining the efficacy of surgery and adjuvant chemoradiation (CRT) versus surgical resection alone in patients with pancreatic adenocarcinoma used 1-to-1 propensity score matching in order to eliminate biases in treatment effects from nonrandom study arm allocation. ${ }^{10}$ After accounting for confounding variables (age, comorbidities, surgical margin, nodal status, etc.) with propensity score analysis, the study concluded that adjuvant CRT improved the overall survival and led to the recommendation for adjuvant CRT in all patients with resected pancreatic adenocarcinoma. While propensity score matching can be an effective method for diminishing treatment selection biases associated with retrospective data, several limitations exist. These include the need for a large control group from which to select appropriate matched pairs and may necessitate the elimination of certain treatment subjects for whom no suitable matched control subject can be identified. These limitations may be particularly notable in HPB surgery, where large numbers of subjects are inherently more difficult to capture as previously discussed. Additionally, significant imbalance in covariates among groups may persist even when implementing a well-devised propensity score model, thus negating its benefits over multivariable analysis.

The use of instrumental variables is an alternative method that addresses another major issue of propensity scoring. The basis of propensity scoring is founded upon observed covariates that must be appropriately identified by researchers and is thus comparable to traditional risk-adjusted analysis. As such, propensity scoring may fail to account for unforeseen confounders that would otherwise be addressed in an RCT design. Instrumental variables are designed to achieve pseudorandomization by accounting for both observed and unobserved confounders, both patient- and provider-specific; in fact, the randomization of RCT design is considered to be a special case of instrumental variable utilization. ${ }^{11}$ To achieve this, instrumental variables must cause substantial variation in the treatment variables affecting outcomes, but cannot themselves have any direct effect on patient outcomes. If the instrumental variable identified fails to produce substantial variation in the treatment variables, then the results will be similar to those produced by conventional regression techniques.
Common examples of instrumental variables utilized in oncologic research include geographic location predicting presentation to high-volume hospitals or year of presentation dictating the standard chemotherapeutic in use (i.e., pre- or postFDA approval) ${ }^{12 \cdot 13}$ In a study ascertaining the effects of pancreatectomy on survival in patients with stage I/II pancreatic adenocarcinoma, McDowell et al. ${ }^{14}$ defined the geographic variation in resection rates as an instrumental variable that itself dictated whether patients received pancreatectomy (i.e., leads to substantial variability in the treatment variable), but in itself had no direct effect on survival and long-term outcomes. Following pseudo-randomization, the study concluded that pancreatectomy was associated with significantly increased survival in patients and thus called for more frequent resection of pancreatic adenocarcinoma in this population. While instrumental variable analysis rivals RCT design in attempting to reconcile treatment selection bias, identification of an appropriate instrumental variable can be difficult and oftentimes can only ascertain treatment effects within a small subset of patient populations. Returning to the McDowell et al. example, ${ }^{14}$ the instrumental variable analysis was utilized specifically for a "marginal" patient population, those for whom the benefits of pancreatectomy are less certain and thus may be subject to the preferred treatment practices of local physicians. This was based on the assumption that excellent and poor operative candidates will unanimously be offered or denied surgery, respectively, regardless of their home regions - in other words, geographic resection rates would not be an applicable instrumental variable in these subpopulations. Instrumental variable analysis can prove an exceptional tool to counter the effects of unforeseen confounding, but the limitations of applicable population analysis must be carefully considered.

Difference-in-difference analysis is a common methodology employed by economists to assess the impacts of new policy implementation in populations exposed to policy changes as compared to unexposed control groups. ${ }^{15}$ The utility of this technique extends beyond the field of econometrics to allow outcomes researchers to determine the effects of changes in healthcare policy or interventions upon exposed patient populations. Ideally, difference-in-difference analysis is most appropriate in determining the causal effects of policy or intervention changes over time. In doing so, it accounts for secular changes, such as outcomes improvement over time or trends in provider utilization of certain techniques, which might otherwise confound their observed impacts. For instance, Loehrer et al. ${ }^{16}$ determined that rates of immediate cholecystectomy for acute cholecystitis varied greatly between self-pay and privately insured patients $(76.8 \%$ and $85.9 \%$, respectively) prior to implementation of the Massachusetts insurance expansion policy in 2006. Following the implementation, a decrease in this disparity was noted among Massachusetts patients - there was a significantly smaller difference in rates of cholecystectomy among self-pay and 
privately insured individuals after policy implementation, suggesting that this policy positively addressed the aforementioned disparity (Fig. 1). ${ }^{16}$ It must be noted that a critical assumption of the difference-in-difference analysis is that of common trends, which supposes that treated and untreated populations would have otherwise continued along the same trajectory over time had the intervention not been implemented. An adequate unexposed control group is therefore necessary to prevent secular confounding by demonstrating steady trends over time in the absence of policy changes. Loehrer et al. ${ }^{16}$ demonstrated unchanging trends of cholecystectomy among patients in control states (not adopting the insurance expansion policy), suggesting that the difference-in-difference observed in Massachusetts was truly the effect of policy change versus some unforeseen secular change (i.e., universally increasing trends towards operative intervention for cholecystitis). Difference-in-difference analysis can provide an excellent method of controlling for secular confounders when retrospectively interpreting the long-term outcomes of policy or interventional changes on patient populations, provided that a suitable control group can be identified.
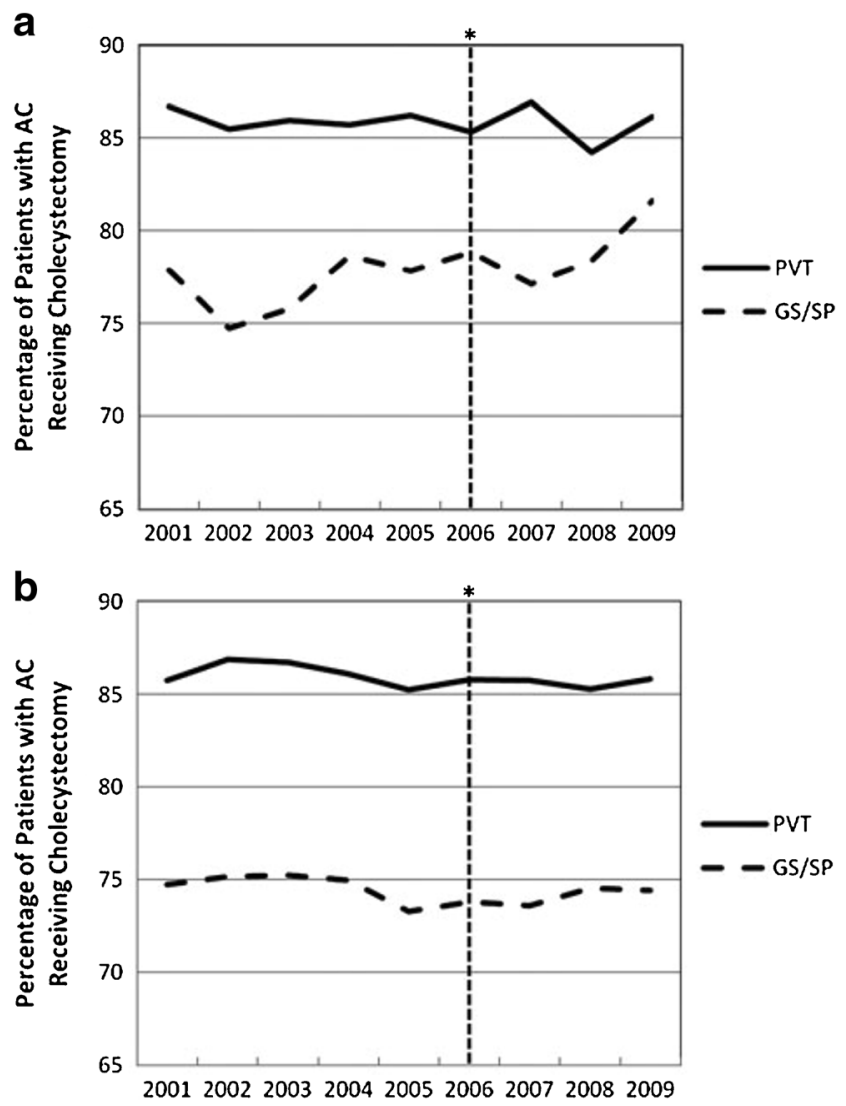

Fig. 1 Unadjusted trends in cholecystectomy for AC by insurance status in Massachusetts (a) and control states (b). Implementation of Massachusetts insurance expansion. PVT indicates private insurance coverage. Taken from Loehrer et al. ${ }^{16}$
Data registries, such as the National Inpatient Sample (NIS); the Surveillance, Epidemiology and End Results (SEER) program; Healthcare Cost and Utilization Project (HCUP); and Medicare, are frequently used to address systems-level questions in outcomes research and the utility of each understandably depends upon the types of questions to be answered. NIS, by capturing hospital discharge data from a $20 \%$ sample of all hospitals participating in the HCUP program across the USA, can be an excellent resource in ascertaining national trends in operative techniques and associated morbidity and mortality. Ejaz et al., ${ }^{17}$ for example, utilized NIS to determine the national trends of open versus laparoscopic hepatic and pancreatic resections and found increasing use of minimally invasive approaches without concomitant increase in morbidity or mortality. A major pitfall of NIS arises from the fact that the hospitals comprising this $20 \%$ national sample change each year, rendering longitudinal studies virtually impossible. As a national registry providing cancer incidence and survival data from registries covering approximately $30 \%$ of the US population, SEER is an exceptional database for assessing trends in oncologic outcomes research - it has, for instance, been used to demonstrate the utility of adjuvant radiotherapy in increasing overall survival for patients with resected pancreatic cancer. ${ }^{18}$ However, while SEER provides adequate data regarding TNM staging and tumor site and morphology, pertinent variables related to the surgical aspects of cancer resection and treatment (tumor margin status, degree of invasion into local structures) are unavailable, thus limiting the depth of surgical outcomes questions that may be answered. Both HCUP and Medicare databases are favored in permitting the performance of longitudinal studies, but both databases are limited in their degree of external validity. While HCUP allows $100 \%$ capture of hospital discharges within a single state, analysis of multiple state registries is required to ascertain national trends. Utilizing Medicare-only data restricts observations to populations greater than 65 years old and thus may not suffice to answer questions regarding surgical diseases or interventions affecting younger cohorts. A common disadvantage among all discussed registries is the lack of granularity in reported variables. One cannot hope to conduct a study similar to Berger et $\mathrm{al}^{4}{ }^{4}$ by implementing these registries, as data regarding pancreatic remnant texture will invariably be missing. Instead, one will find that data registries are ideal for investigating broader issues, such as examining national trends in regionalization of complex cancer surgery. ${ }^{19}$

Quality collaboratives and multicenter consortiums are alternative databases that may prove more useful to address novel questions requiring a higher degree of granularity pertaining to the field of surgery. The American College of Surgeons National Surgical Quality Improvement Program (ACS-NSQIP) is a well-known quality collaborative specifically focusing on amassing data regarding surgical outcomes 
and complications from hundreds of hospitals throughout the nation. Surgery-specific variables, such as operative duration and postoperative complications (surgical site infections, sepsis, deep venous thrombosis/pulmonary embolism, transfusions, re-operation, etc.), are perhaps the greatest benefit of utilizing a database such as ACS-NSQIP for surgical outcomes research. To demonstrate, Newhook et al. ${ }^{20}$ used ACS-NSQIP data to show that patients undergoing PD for benign and premalignant lesions exhibited higher rates of organ space infection and sepsis versus those undergoing malignant resection, thus suggesting that more careful consideration of PD in these patients should be employed. While ACSNSQIP may lack a certain degree of granularity with respect to surgical subspecialties such as HPB, current efforts are focused on piloting specialty-specific collaboratives to address such issues. The ACS-NSQIP Pancreatectomy Demonstration Project, for instance, seeks to provide a multitude of HPB-specific variables (preoperative biliary stent placement, pancreatic duct diameter and remnant gland texture, anastomotic technique and vascular resection, drain placement and amylase levels, rates of PF, and delayed gastric emptying (DGE)) so that outcomes researchers can address very precise questions on a much broader scale. ${ }^{21-23}$ As an example, this pilot database has been utilized by Parmar et al. ${ }^{24}$ to identify $\mathrm{PF}$ formation, postoperative sepsis and reoperation as independent predictors of DGE following PD or total pancreatectomy. Similarly, multicenter consortiums are primed tools for investigating questions related to subspecialty-specific surgical outcomes and harbor the added benefit of promoting widespread collaboration and idea sharing among various facilities. Constructed at the individual hospital level, multicenter consortiums have the granular data necessary to investigate specialty-specific research questions. As an example, Ahmad et al. ${ }^{25}$ conducted a multicenter study among six institutions revealing PF to be predictive of readmission and infection, nutritional status, and DGE to be the most frequent reasons for readmission following PD. While quality collaboratives and multicenter consortiums undeniably provide researchers with data that are imperative to assessing to surgical outcomes, both databases are logistically difficult and expensive to coordinate. ACS-NSQIP requires the hiring of a trained surgical clinical reviewer for each participating hospital to collect pertinent data from the preoperative to 30 -day postoperative periods. Organizing multicenter consortiums are an even more daunting task when considering the need for full collaboration between hospitals and approval among multiple Institutional Review Boards.

Our framework has sought to demonstrate the intricacy and complexity of the outcomes equation. Treatment is but one piece of the puzzle - the other pieces must be gathered and considered in their respective places or roles in order to fully grasp the entire picture that is a patient's outcome. Furthermore, by outlining the utility and limitations of various healthcare databases and analytical methods, we have demonstrated how outcomes research can help to identify and subsequently answer novel clinical questions that have thus far eluded our investigative abilities. In many ways, advancing our understanding of patient outcomes beyond the limits of treatment effects and overcoming the inherent power limitations of RCT design are the hallmarks that render outcomes research indispensible to the field of surgery.

\section{Compliance with Ethical Standards}

Funding No grants or other forms of funding or assistance were utilized in the formation of this manuscript.

\section{References}

1. Kaido T. Randomized controlled trials on hepato-biliary-pancreatic surgery. J Hepatobiliary Pancreat Surg 2004;11:381-389.

2. Hodgson DC, Fuchs CS, Ayanian JZ. Impact of patient and provider characteristics on the treatment and outcomes of colorectal cancer. J Natl Cancer Inst 2001;93:501-515.

3. Lee SJ, Earle CC, Weeks JC. Outcomes research in oncology: history, conceptual framework and trends in the literature. J Natl Cancer Inst 2000;92:195-204.

4. Berger AC, Howard TJ, Kennedy EP, Sauter PK, Bower-Cherry M, Dutkevitch S, Hyslop T, Schmidt CM, Rosato EL, Lavu H, Nakeeb A, Pitt HA, Lillemoe KD, Yeo CJ. Does type of pancreaticojejunostomy after pancreaticoduodenectomy decrease rate of pancreatic fistula? A randomized, prospective, dualinstitution trial. J Am Coll Surg 2009;208:738-749.

5. Lin JW, Cameron JL, Yeo CJ, Riall TS, Lillemoe KD. Risk factors and outcomes in postpancreaticoduodenectomy pancreaticocutaneous fistula. J Gastrointest Surg 2004;8:951-959.

6. Relles DM, Richards NG, Bloom JP, Kennedy EP, Sauter PK, Leiby BE, Rosato EL, Yeo CJ, Berger AC. Serum blood urea nitrogen and serum albumin on the first postoperative day predict pancreatic fistula and major complications after pancreaticoduodenectomy. J Gastrointest Surg 2013;17:326-331.

7. Birkmeyer JD, Siewers AE, Finlayson EV, Stukel TA, Lucas FL, Batista I, Welch HG, Wennberg DE. Hospital volume and surgical mortality in the United States. N Engl J Med 2002;346:1128-1137.

8. Michalski CW, Wietz J, Buchler MW. Surgery insight: surgical management of pancreatic cancer. Nat Clin Pract Oncol 2007;4: 526-535.

9. Newgard CD, Hedges JR, Arthur M, Mullins RJ. Advanced statistics: the propensity score - a method for estimating treatment effect in observational research. Acad Emerg Med 2004;11:953-961.

10. Hsu CC, Herman JM, Corsini MM, Winter JM, Callister MD, Haddock MG, Cameron JL, Pawlik TM, Schulick RD, Wolfgang CL, Laheru DA, Farnell MB, Swartz MJ, Gunderson LL, Miller RC. Adjuvant chemoradiation for pancreatic adenocarcinoma: the Johns Hopkins Hospital-Mayo Clinic collaborative study. Ann Surg Oncol 2010;17:981-990.

11. Newhouse JP, McClellan M. Econometrics in outcomes research: the use of instrumental variables. Annu Rev Publ Health 1998;19: 17-34.

12. Pezzin LE, Laud P, Yen TWF, Neuner J, Nattinger AB. Reexamining the relationship of breast cancer hospital and surgical 
volume to mortality: and instrumental variable analysis. Med Care 2015;Epub ahead of print.

13. Mack CD, Brookhart MA, Glynn RJ, Meyer AM, Carpenter WR, Sandler RS, Stürmer T. Comparative effectiveness of oxaliplatin versus 5-flurouracil in older adults: an instrumental variable analysis. Epidemiology 2015;26:690-699.

14. McDowell BD, Chapman CG, Smith BJ, Button AM, Chrischilles EA, Mazhir JJ. Pancreatectomy predicts improved survival for pancreatic adenocarcinoma: results of an instrumental variable analysis. Ann Surg 2015;261:740-745.

15. Wooldridge JM. Econometric Analysis of Cross Section and Panel Data. Cambridge, MA: The MIT Press; 2001.

16. Loehrer AP, Song Z, Auchincloss HG, Hutter MH. Influence of health insurance expansion on disparities in the treatment of acute cholecystitis. Ann Surg 2015;262:139-145.

17. Ejaz A, Sachs T, He J, Spolverato G, Hirose K, Ahuja N, Wolfgang CL, Makary MA, Weiss M, Pawlik TM. A comparison of open and minimally invasive surgery for hepatic and pancreatic resections using the nationwide inpatient sample. Surgery 2014;156:538-547.

18. Opfermann KJ, Wahlquist AE, Garrett-Mayer E, Shridhar R, Cannick L, Marshall DT. Adjuvant radiotherapy and lymph node status for pancreatic cancer: results of a study from the surveillance, epidemiology, and end results (SEER) registry data. Am J Clin Oncol 2014;37:112-116.

19. Stitzenberg KB, Meropol NJ. Trends in centralization of cancer surgery. Ann Surg Oncol 2010;17:2824-2831.
20. Newhook TE, LaPar DJ, Lindberg JM, Bauer TW, Adams RB, Zaydfudim VM. Morbidity and mortality of pancreaticoduodenectomy for benign and premalignant pancreatic neoplasms. J Gastrointest Surg 2015;19:1072-1077.

21. Lee CW, Pitt HA, Riall TS, Ronnekleiv-Kelly SS, Israel JS, Leverson GE, Parmar AD, Kilbane EM, Hall BL, Weber SM. Low drain fluid amylase predicts absence of pancreatic fistula following pancreatectomy. J Gastrointest Surg 2014;18:1902-1910.

22. Beane JD, House MG, Pitt SC, Kilbane EM, Hall BL, Parmar AD, Riall TS, Pitt HA. Distal pancreatectomy with celiac axis resection: what are the added risks? HPB (Oxford) 2015;17:777-784.

23. Tamirisa NP, Parmar AD, Vargas GM, Mehta HB, Kilbane EM, Hall BL, Pitt HA, Riall TS. Relative contributions of complications and failure to rescue on mortality in older patients undergoing pancreatectomy. Ann Surg 2015;Epub ahead of print.

24. Parmar AD, Sheffield KM, Vargas GM, Pitt HA, Kilbane EM, Hall BL, Riall TS. Factors associated with delayed gastric emptying after pancreaticoduodenectomy. HPB (Oxford) 2013;15:763-772.

25. Ahmad SA, Edwards MJ, Sutton JM, Grewal SS, Hanseman DJ, Maithel SK, Patel SH, Bentram DJ, Weber SM, Cho CS, Winslow ER, Scoggins CR, Martin RC, Kim HJ, Baker JJ, Merchant NB, Parikh AA, Kooby DA. Factors influencing readmission after pancreaticoduodenectomy: a multi-institutional study of 1302 patients. Ann Surg 2012;256:529-537. 